Trauma Berufskrankh 2011 13:233 DOI 10.1007/s10039-011-1814-0

Online publiziert: 26. November 2011

๑) Springer-Verlag 2011

\author{
P.A. Grützner \\ BG-Unfallklinik Ludwigshafen
}

\title{
Minimalinvasive Operationsverfahren
}

gibt es auch relevante objektive Parameter wie einen deutlich geringeren Blutverlust. Solche neuen Operationsverfahren werden jedoch in der Regel erst durch die Entwicklung von neuen Implantaten und Instrumentarien realisierbar. Die Versorgung der Akromioklavikulargelenksprengung mittels TightRope ${ }^{\oplus}$ ist ein solches Beispiel. Hier wird uns erstmalig durch das neue Implantat nicht nur die Change zur Minimalinvasivität geboten, sondern dem Patienten aufgrund der nicht notwendigen Materialentfernung auch eine zweite Operation erspart. Ob die Ergebnisse allerdings wesentlich besser als die der offenen Technik mit Stabilisation mittels Zuggurtung oder auch Hakenplatte sind, muss durch wissenschaftliche Arbeiten erst noch belegt werden.

Wenn große Zugänge vermieden werden wollen, die Manipulation im dann nicht einsehbaren Operationsgebiet aber trotzdem notwendig ist, sind spezielle Instrumente sehr hilfreich. Manchmal kann man sich aber auch einfach mit dem bestehenden Inventar behelfen, wie im Beitrag Tipps \& Tricks anschaulich belegt. Man muss nur wissen wie und im Fall der Fälle auch dran denken. Ansonsten stehen zur direkten Visualisierung zunehmend endoskopische Verfahren zur Verfügung, zur indirekten Darstellung anatomischer Strukturen moderne bildgebende Systeme, die zusätzliche Navigationsmöglichkeiten bieten. Die perkutan navigierte Schraubenosteosynthese am Azetabulum oder die Iliosakralfugenverschraubung unter Anwendung eines 3D-Bildwandlers sind beispielhaft dafür, wie die Integration von moderner Technologie dem minimalinvasiven Vorgehen den Boden ebnet.

Neben Standortbestimmungen durch die Artikel der Rubrik Standards in der
Unfallchirurgie: Arthroskopische Verfahren soll Ihnen dieses Heft anhand von ausgewählten Beiträgen beispielhaft den aktuellen Stand der minimalinvasiven Verfahren auf dem Gebiet der Unfallchirurgie und Orthopädie näherbringen. An dieser Stelle gilt es, den Autoren für ihre engagierten, kurzweiligen und trotzdem sehr informativen Beiträge zu danken.

Viel Vergnügen und Wissensgewinn bei der Lektüre.

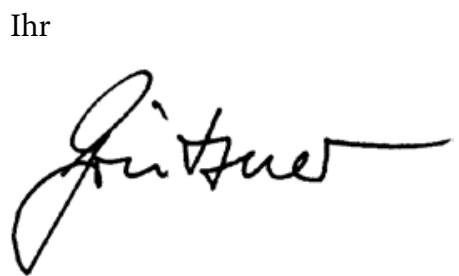

Prof. Dr. P.A. Grützner

\section{Korrespondenzadresse \\ Prof. Dr. P.A. Grützner \\ BG-Unfallklinik Ludwigshafen, Ludwig-Guttmann-Straße 13, 67071 Ludwigshafen \\ gruetzner@bgu-ludwigshafen.de}

